In phase two we analyzed field notes, focus group transcriptions, and post-screening and 6-month follow-up surveys. In both phases, we found that documentary video effectively educated viewers and initiated discussion. Further, results from our extended data collection and analysis in phase two suggests that individuals were impacted along a scale: education, knowledge diffusion (sharing), and/or action to improve circumstances. We also offer insight on strategies to move research-evidence from discussion to implementation.

\section{EVERYTHING IN MODERATION: INTERACTION EFFECTS AMONG GENDER, RACE, AND DRIVING REDUCTION/CESSATION}

J. Vivoda ${ }^{1}$, C.M. Connell², A. Schulz², J. Grengs², S. Heeringa ${ }^{2}$, 1. Sociology and Gerontology, Miami University, Oxford, Ohio, 2. University of Michigan, Ann Arbor, Michigan

This research explored the moderating effect of the transportation environment on the relationships among gender, race, and driving reduction and cessation (DRC). We hypothesized that the higher likelihood of DRC among women and racial minorities may be partially explained by high congestion/roadway density. Models fully interacted by gender and race were also fit to explore whether the relationships among DRC and the other covariates were differentially affected by these demographics.

The transportation environment was operationalized using a measure of roadway density calculated from Geographic Information Systems data, and with congestion data from the Urban Mobility Scorecard. Seven waves of data from the Health and Retirement Study were combined with this environmental data, and discrete time survival analysis techniques were used in the analysis; driving reduction (DR) and driving cessation (DC) were analyzed as separate outcomes.

A significant interaction was observed between roadway density and gender in the model assessing DR (higher density only affected men), but not in the DC model. This was counter to our original hypothesis, and may be related to the decreased likelihood of men to change their driving habits overall. Interactions between the transportation environment and race were not statistically significant in the DR models, but were significant in the models assessing DC (White and Hispanic older adults had higher odds of DC given more congestion). The models fully interacted by gender and race also revealed several additional significant interactions among other covariates in the models and DRC, including relationship status, age, education, and household size.

\section{THE CREATION OF AGE-FRIENDLY ENVIRONMENTS IS ESPECIALLY IMPORTANT TO FRAIL OLDER PEOPLE}

H. van Dijk, A.P. Nieboer, J. Cramm, Institution of Health

Policy \& Management, Erasmus University Rotterdam, Rotterdam, Zuid-Holland, Netherlands

Worldwide efforts are being made to reduce reliance on expensive long-term care, resulting in a shift toward deinstitutionalisation and ageing in place. However, we lack insight into whether neighbourhoods are able to meet both frail and non-frail older people's environmental needs. Based on the WHO framework for age-friendly cities, this study aimed to characterise the relationship between frailty and ageing in place, and to identify differences in neighbourhood characteristics supporting ageing in place missed by frail and non-frail older people. A concurrent nested mixed-methods approach was used. For quantitative evaluation, a sample of 945 independently living older adults residing in four districts of Rotterdam was asked to complete a questionnaire in 2013 [response rate, $62 \%(n=558)$ ]. In addition, 32 qualitative interviews were conducted with frail and non-frail older people. The results demonstrated that gender, age, and especially frailty were related to missed neighbourhood characteristics. Qualitative data showed that older people display awareness of their increasing frailty and often acknowledge that it increased their needs for neighbourhood characteristics enabling them to age in place. Thus, this study supported our expectation that the person-environment fit is not static. Expectations regarding neighbourhood characteristics seem to dissipate with advanced age and increasing frailty. Diversity in frailty level, gender, and age, but also the interrelatedness of age and frailty, should be accounted for in the identification of social and physical neighbourhood characteristics that community-dwelling older adults need to age in place.

\section{DISCREPANCY OF SELF-ASSESSMENTS AND STANDARDIZED TESTS IN VISION/HEARING ABILITIES IN THE ELDERLY}

G.G. Haanes ${ }^{1}$, G. Eilertsen ${ }^{2}$, 1. Nursing, University of Faroe Islands, Tórshavn, Faroe Islands, 2. University College of South East Norway, Drammen, Norway

Aim: To investigate whether answers provided by older home-care recipients to the question "Do you think your vision/hearing is good (0), not so good (1), poor (2), or very poor (3)?" can be used to identify those who have vision and hearing problems, and whether these elderly can provide a valid self-report of their vision and hearing.

Methods: Receiving operating characteristic (ROC) analysis was used to compare self-evaluations of vision and hearing with those yielded by a gold-standard test. The vision and hearing performances of 93 people, aged $\geq 80$ years in the home-care setting were screened with a LogMAR chart and a portable pure-tone audiometer and a self-assessment screen.

Results: Comparisons of the findings using the cutoff point on the self-assessment scale with those of the goldstandard tests, yielded 40 false negatives for vision and 18 false negatives for hearing, indicating that a significant proportion of older people report their vision and hearing abilities as being good when standardized tests indicate that they are not.

Conclusion: Area under the ROC curve for self-assessment of vision was $69 \%$, indicating the self-assessment question was a poor test. Area under the ROC curve for selfassessment of hearing was $73 \%$, which may be considered a "fair" test. However, hearing self-evaluations are still insufficient for deciding who should be referred to a specialist for hearing examination.

\section{HOME MODIFICATION PROGRAM CHALLENGES: BALANCING BEHAVIORS, PREFERENCES, NEEDS, AND COST}

N. Brossoie, Center for Gerontology, Virginia Polytechnic Institute \& State University, Blacksburg, Virginia 